\title{
Light-curing units used in dentistry: Effect of their characteristics on temperature development in teeth
}

\author{
Mathieu MOUHAT ${ }^{1}$, Lina STANGVALTAITE-MOUHAT ${ }^{1,2}$, James MERCER ${ }^{3}$, Bo Wold NILSEN ${ }^{1}$ \\ and UIf ÖRTENGREN 1,4
}

\author{
${ }^{1}$ Department for Clinical Dentistry/Faculty of Health Sciences, UiT the Arctic University of Norway, Hansine Hansens veg 86, 9019 Tromsø, Norway \\ ${ }^{2}$ Oral Health Center of Expertise in Eastern Norway, Sørkedalsveien 10A, 0369 Oslo, Norway \\ ${ }^{3}$ Department of Medical Biology/Faculty of Health Sciences, UiT the Arctic University of Norway, 9037 Troms $\varnothing$, Norway \\ ${ }^{4}$ Department of Cariology, Institute of Odontology/Sahlgrenska Academy, University of Gothenburg, Medicinargatan $12 E, 41390$ Gothenburg, \\ Sweden \\ Corresponding author, Mathieu MOUHAT; E-mail: mathieu.mouhat@uit.no
}

\begin{abstract}
This study aimed to investigate pulp chamber and surface temperature development using different LED light curing units (LCUs). Eight brands of LED-LCUs were tested in a laboratory bench model. The pulp chamber and surface temperature were recorded with a type $\mathrm{T}$ thermocouple and infrared cameras, respectively. The highest pulp chamber and surface temperature increase was $6.1 \pm 0.3^{\circ} \mathrm{C}$ and $20.1 \pm 1.7^{\circ} \mathrm{C}$, respectively. Wide-spectrum LED-LCUs produced higher pulp chamber temperature increase at $0 \mathrm{~mm}$ and $2 \mathrm{~mm}$ but lower at $4 \mathrm{~mm}$. Narrow-spectrum LED-LCUs produced higher surface temperature increase. LED-LCU featuring modulated output mode resulted in lower increase in pulp chamber temperature but higher on surface temperature. LED-LCU with light guide tip delivering an inhomogeneous beam caused higher increase in temperature on the surface and in the pulp chamber. LED-LCUs with different spectral emission, output mode and light guide tip design contributed to different temperature development in the pulp chamber and at the surface of teeth.
\end{abstract}

Keywords: LED dental curing light, Spectral emission, Output mode, Pulp chamber, Temperature

\section{INTRODUCTION}

Light-cured resin-based composites (RBC) are extensively used worldwide as a material for direct and indirect restorations ${ }^{1}$. Light emitting diodes light curing units (LED-LCUs) are currently the device of choice for practitioners to cure RBC. It has been shown that light curing of RBC is more complicated than most users assume, a fact that is often underrated by the dental community $^{2-4)}$. Inappropriate use of LED-LCUs will lead to poorly cured RBC, which may increase elution of unreacted substances, and increased rate of restoration failure due to secondary caries or mechanical failure ${ }^{5,6}$. In order to prevent under-curing of $\mathrm{RBC}$, it has been proposed to increase the curing time (CT) beyond the recommendation made by the manufacturers ${ }^{7}$. However, in vitro studies suggested that longer $\mathrm{CT}$, higher radiant emittance, and closer LED-LCUs' light guide tip distance from the tooth surface may cause thermal injury to the pulp and the surrounding oral soft tissue ${ }^{8,9}$. A recent in vivo study confirmed previous in vitro findings that longer exposure time and higher radiant emittance of LED-LCU are responsible for higher pulpal temperature rise. It has been shown that a $60 \mathrm{~s}$ exposure time with a wide-spectrum LED-LCU reached a pulpal temperature rise higher than $5.5^{\circ} \mathrm{C}^{10)}$, a threshold value considered harmful for the pulp ${ }^{11}$. The use of LED-LCUs and the heat generated might harm soft oral tissues as well.

Color figures can be viewed in the online issue, which is available at J-STAGE.

Received Aug 18, 2020: Accepted Jan 26, 2021

doi:10.4012/dmj.2020-305 JOI JST.JSTAGE/dmj/2020-305
Clinical cases of burning sensation of the lips of patients have been reported during light-curing with LED-LCUs even though the rubber dam was in position ${ }^{12}$. An in vivo study performed on swine gingiva that investigated the temperature increase on the gingival tissue when using a wide-spectrum LED-LCU reported that the use of rubber dam did not prevent the temperature rise and gingival lesions ${ }^{13)}$. While the exothermic reaction of the RBC might also have an impact on pulp chamber temperature rise, the irradiance from LED-LCU seems to remain the main factor responsible for temperature increase in the pulp chamber and at the surface of teeth $^{14)}$. Apart from CT, distance and radiant emittance, there are indications that the temperature development might also depend on LED-LCU spectral emission, output mode and light guide tip design ${ }^{15-17)}$.

LED-LCUs with broader wavelength spectral emission are known under different names as polywave ${ }^{\circledR}$, multi-wave, multi-peak and broad banded ${ }^{18}$. A benefit of their broader spectra is their ability to activate other photoinitiators (e.g. phenyl-propanedione, Lucirin ${ }^{\circledR}$ TPO and Ivocerin ${ }^{\circledR}$ ) in addition to the most widely used camphorquinone and therefore initiate photopolymerization of a wider range of $\mathrm{RBC}^{19,20)}$. LEDLCUs with broader wavelength spectral emission (wide-spectrum) have both a "violet" light (380-425 nm with a peak at $\sim 405 \mathrm{~nm})$ and a "blue" light (425-500 $\mathrm{nm}$ with a peak at $\sim 455 \mathrm{~nm}$ ), while narrow-spectrum LED-LCUs have only one peak emission at $\sim 455 \mathrm{~nm}$ or at $\sim 477 \mathrm{~nm}$ which covers the wavelength of "blue" 
light ${ }^{16)}$. Wide-spectrum LED-LCUs have been shown to lack uniformity in radiant emittance and spectral emission when measured at their end light guide tip using a laser beam profiler camera and an integrating sphere ${ }^{21,22)}$. Many LED-LCUs offer different output modes that can be commonly grouped as continuous and discontinuous light curing techniques; discontinuous further being divided into output modes as soft start mode and modulated output mode (in the literature also called ramp, pulse and step) ${ }^{15,23)}$. It has been stated that modulated output mode provided clinical benefits such as decreased polymerization shrinkage and reduced adverse heating effects ${ }^{24,25)}$. It has also been suggested that the irradiance inhomogeneity of LED-LCUs might be a factor contributing to different temperature development at a tooth surface and in a pulp chamber ${ }^{26,27)}$. It has been shown that the light guide design of LEDLCU is of importance; different light guides were used on the same LED-LCU core unit and yielded different irradiance and beam homogeneity values which may result in different temperature development ${ }^{28)}$.

In a previous study from our group, two different brands of LED-LCUs, both wide-spectrum, reached a maximum temperature in a pulp chamber and on the tooth surface of $43.1 \pm 0.9^{\circ} \mathrm{C}$ and $58.1 \pm 0.9^{\circ} \mathrm{C}$, respectively ${ }^{8}$. Time was the most influential factor for temperature development in the pulp chamber, while irradiance was the strongest influencing factor on surface temperatures. To the best of the authors' knowledge no studies investigating the combined effect of irradiance, time, distance, spectral emission, output mode and light guide tip design simultaneously on temperature development at a tooth surface and in its pulp chamber have been previously performed. The aim of the present study was therefore to investigate and compare temperature development on the tooth surface and in the pulp chamber of a natural tooth with different brands of LED-LCUs using a laboratory bench model we have described in a previous study ${ }^{8}$. Of particular interest in this study was the influence of the spectral emission, output mode and the light guide tip design of the LED-LCUs. The null hypothesis was that there were no differences in the temperature development (on the tooth surface and in the pulp chamber) when exposed to LED-LCUs having different spectral emission, output mode and light guide tip design. In addition, separately for narrow- and wide-spectrum LED-LCUs, we investigated the adjusted effect of irradiance, time, distance, output mode and light guide tip design on the temperature development on the tooth surface and in the pulp chamber.

\section{MATERIALS AND METHODS}

Spectral emission, output mode and light guide tip design characterization of the LED-LCU tested

Eight different brands of LED-LCUs with different spectral emission, output mode and light guide tip design were tested: Bluephase Style $^{\circledR}$ (battery and mains powered), Bluephase $\mathrm{G} 2^{\circledR}$ in two modes (High and
Low mode) (Ivoclar Vivadent, Schaan, Lichtenstein), TransLux $^{\circledR}$ Wave and TransLux ${ }^{\circledR}$ 2Wave (Kulzer, Hanau, Germany), Elipar ${ }^{\mathrm{tm}}$ DeepCure and Elipar ${ }^{\mathrm{tm}}$ S10 (3M ESPE, Seefeld, Germany), SmartLite ${ }^{\circledR}$ Focus (Dentsply Caulk, Milford, CT, USA) and Demitm Ultra (Kerr Dental, Orange, CA, USA) (Table 1). The spectral emission (narrow-spectrum, wide-spectrum (Fig. 1A), output mode (modulated, continuous) (Fig. 1B) and irradiance of each LED-LCU was measured five times using a calibrated laboratory-grade NIST-referenced USB4000 spectrometer (Managing Accurate Resin Curing (MARC) System; Bluelight Analytics, Halifax, Canada) (Table 1). The radiant exposure, which is the product of irradiance and time expressed in Joule per square centimeter $\left(\mathrm{J} / \mathrm{cm}^{2}\right)$, was calculated for each LEDLCU at $0 \mathrm{~mm}, 2 \mathrm{~mm}$ and $4 \mathrm{~mm}$ distance with $10 \mathrm{~s}$ and $20 \mathrm{~s}$ CT (Table 2). The light guide tip design was investigated with a liquid crystal sheet (\#61161, Edmunds Optics, Barrington, NJ, USA) to visualize the heat distribution pattern (Fig. 1C). The liquid crystal sheet contains heat-sensitive liquid crystals, having optical properties similar to a crystalline solid and indicate change in temperature by changing colors ${ }^{29)}$. The light guide tip of each LED-LCU was placed in direct contact on the back side of a thin liquid crystal sheet and the resulting heat induced changes in the thermal pattern of the liquid crystals was observed on the opposite side. The thermal pattern was photographed with a camera (Canon EOS 700D, Canon, Tokyo, Japan) with a macro lens (SP 90 mm F/2.8 Macro VC, Tamron, Saitama, Japan) after $1 \mathrm{~s}$ of direct contact. The LED-LCU light guide tip was considered as inhomogeneous when more than one thermal hot spot appeared with gaps in between them with no temperature development, for example as shown in Fig. 1, C1ii. When there was one single thermal hot spot, the LED-LCU light guide tip was considered as homogenous, for example as shown in Fig. 1, C2ii. Based on the photographs and these criteria, the light guide tip was defined as delivering homogeneous beam (light guide tip homogeneous) and inhomogeneous beam (light guide tip inhomogeneous). The LED-LCU Bluephase Style ${ }^{\circledR}$ was tested with an older light guide tip design where the three separated LED chips were clearly visible through the light guide tip (Bluephase Style ${ }^{\circledR}$ battery) (Fig. 1, C1i.) and a newer light guide tip design where the LED chips were less clearly visible (Bluephase Style ${ }^{\circledR}$ mains powered) (Fig. 1, C2i.). It should be noted that the bottom left-hand corner chip of the LED-LCU with an older light guide tip design emitting shorter emission wavelength ("violet" light) displayed a thermal spot with lower temperature compared to the two other thermal spots emitting longer emission wavelength ("blue" light) (Fig. 1, C1ii.).

\section{Tooth preparation and thermal environment control}

A caries-free extracted human molar no older than 6 months was used to assess heat development on the surface and in the pulp chamber. The tooth was stored in $0.5 \%$ Chloramine-T solution according to ISO/TS $11405-2015$ in a refrigerator $\left(4 \pm 1^{\circ} \mathrm{C}\right)$ prior to use and 
Table 1 Description of LED light curing units investigated in the present study

\begin{tabular}{|c|c|c|c|c|c|c|}
\hline $\begin{array}{l}\text { LED Light } \\
\text { curing unit }\end{array}$ & Manufacturer & $\begin{array}{l}\text { Spectral } \\
\text { emission }\end{array}$ & Output mode & $\begin{array}{l}\text { Light guide tip } \\
\text { design }\end{array}$ & $\begin{array}{l}\text { Wavelength } \\
\quad(\mathrm{nm})\end{array}$ & $\begin{array}{c}\text { Mean }(\mathrm{SD}) \\
\text { irradiance } \\
(0 \mathrm{~mm}) \mathrm{mW} / \mathrm{cm}^{2}\end{array}$ \\
\hline $\begin{array}{l}\text { Bluephase } \\
\text { Style }^{\circledR} \text { battery }\end{array}$ & $\begin{array}{l}\text { Ivoclar Vivadent, } \\
\text { Schaan, Lichtenstein }\end{array}$ & $\begin{array}{l}\text { Wide- } \\
\text { spectrum }\end{array}$ & Continuous & $\begin{array}{l}\text { Inhomogeneous } \\
\text { beam }\end{array}$ & $385-515$ & $1,284(110)$ \\
\hline $\begin{array}{l}\text { Bluephase } \\
\text { Style }^{\circledR} \text { mains }\end{array}$ & Ivoclar Vivadent & $\begin{array}{l}\text { Wide- } \\
\text { spectrum }\end{array}$ & Continuous & $\begin{array}{l}\text { Homogeneous } \\
\text { beam }\end{array}$ & $385-515$ & $1,260(38)$ \\
\hline $\begin{array}{l}\text { Bluephase } \mathrm{G} 2^{\circledR} \\
\text { High mode }\end{array}$ & Ivoclar Vivadent & $\begin{array}{l}\text { Wide- } \\
\text { spectrum }\end{array}$ & Continuous & $\begin{array}{l}\text { Homogeneous } \\
\text { beam }\end{array}$ & $385-515$ & $1,455(60)$ \\
\hline $\begin{array}{l}\text { Bluephase } \mathrm{G} 2^{\circledR} \\
\text { Low mode }\end{array}$ & Ivoclar Vivadent & $\begin{array}{l}\text { Wide- } \\
\text { spectrum }\end{array}$ & Continuous & $\begin{array}{l}\text { Homogeneous } \\
\text { beam }\end{array}$ & $385-515$ & $808(45)$ \\
\hline $\begin{array}{l}\text { TransLux } \\
\text { 2Wave }\end{array}$ & $\begin{array}{l}\text { Kulzer, Hanau, } \\
\text { Germany }\end{array}$ & $\begin{array}{l}\text { Wide- } \\
\text { spectrum }\end{array}$ & Continuous & $\begin{array}{l}\text { Homogeneous } \\
\text { beam }\end{array}$ & $385-510$ & $1,334(26)$ \\
\hline $\begin{array}{l}\text { TransLux } \\
\text { Wave }\end{array}$ & Kulzer & $\begin{array}{l}\text { Narrow- } \\
\text { spectrum }\end{array}$ & Continuous & $\begin{array}{l}\text { Homogeneous } \\
\text { beam }\end{array}$ & $440-480$ & $1,486(160)$ \\
\hline $\begin{array}{l}\text { Elipar }^{\mathrm{tm}} \\
\text { DeepCure }\end{array}$ & $\begin{array}{l}\text { 3M ESPE, St. Paul, } \\
\text { MN, USA }\end{array}$ & $\begin{array}{l}\text { Narrow- } \\
\text { spectrum }\end{array}$ & Continuous & $\begin{array}{l}\text { Homogeneous } \\
\text { beam }\end{array}$ & $430-480$ & $2,028(251)$ \\
\hline Elipar $^{\mathrm{tm}}$ S10 & 3M ESPE & $\begin{array}{l}\text { Narrow- } \\
\text { spectrum }\end{array}$ & Continuous & $\begin{array}{l}\text { Homogeneous } \\
\text { beam }\end{array}$ & $430-480$ & $1,838(243)$ \\
\hline $\begin{array}{l}\text { SmartLite }^{\circledR} \\
\text { Focus }\end{array}$ & $\begin{array}{l}\text { Dentsply Caulk, } \\
\text { Milford, DE, USA }\end{array}$ & $\begin{array}{l}\text { Narrow- } \\
\text { spectrum }\end{array}$ & Continuous & $\begin{array}{l}\text { Homogeneous } \\
\text { beam }\end{array}$ & $460-490$ & $1,079(80)$ \\
\hline Demi $^{\text {tm }}$ Ultra & $\begin{array}{l}\text { Kerr Dental, } \\
\text { Orange, CA, USA }\end{array}$ & $\begin{array}{l}\text { Narrow- } \\
\text { spectrum }\end{array}$ & Modulated & $\begin{array}{l}\text { Homogeneous } \\
\text { beam }\end{array}$ & $450-470$ & $1,262(258)$ \\
\hline
\end{tabular}

in-between the experiments. The cusps were cut with a diamond saw (Accutom 50, Struers, Ballerup, Denmark) to create a flat dentine surface. The remaining dentine thickness was $0.6 \mathrm{~mm}$ as determined from an intraoral radiograph (Planmeca Intra X-ray unit with Romexis, Planmeca Oy, Helsinki, Finland). The root was cut around $0.5 \mathrm{~mm}$ from the apex and a thin calibrated thermocouple (Type T copper constantan) for measuring the temperature within the pulp chamber was inserted as close as possible to the pulpal horn under radiographic control. Temperature changes were continuously recorded with a data logger (OQ610 temperature logger, Grant Instruments, Cambridge, UK) using the software SquirrelView (version 3.9, Grant Instruments) connected to a standard desk-top computer. In order to simulate the environmental conditions within the oral cavity, the tooth was securely seated in a suitably sized hole cut in a thin plastic sheet, with the root protruding out on one side of the hole and the coronal part on the opposite side. The plastic sheet with the tooth was placed in a thermostatically controlled and circulated water bath maintained at $37 \pm 1^{\circ} \mathrm{C}$ with the root being immersed in the water up to the level of the cemento-enamel junction and the coronal part in the air. The tooth surface temperature was measured with calibrated infrared cameras (Thermacam S65 HS and Thermacam SC645, Flir Systems, Wilsonville, OR, USA). The thermal emissivity was set at 0.98 . The entire setup is described in detail in our previous study ${ }^{8}$. All temperatures were measured to an accuracy of $0.1^{\circ} \mathrm{C}$. With this setup the baseline temperature was on average $35.9 \pm 0.3^{\circ} \mathrm{C}$ in the pulp chamber and $33.4 \pm 0.8^{\circ} \mathrm{C}$ at the tooth surface. The room temperature during the experiment was measured to be $21 \pm 1^{\circ} \mathrm{C}$. Figure 2 describes our experimental setup.

For the temperature development on the surface and in the pulp chamber the measurements followed the same protocol as in our previous study; five measurements with each LED-LCU at each case occasion were performed at $0 \mathrm{~mm}, 2 \mathrm{~mm}$ and $4 \mathrm{~mm}$ distance having $10 \mathrm{~s}$ and $20 \mathrm{~s} \mathrm{CT}$. The distances chosen were based on distances of the LED-LCU tip to the tooth generally used in a clinical setting ${ }^{15)}$.

\section{Ethical permission}

Since the study involved the use of an extracted human tooth, we applied to the Norwegian Regional Committee for Medical and Health Research Ethics (REK) for permission to carry out the study. The human material (tooth) being anonymized, they concluded that such permission was not necessary (2015/234/REK Nord).

\section{Statistics}

The data was analyzed using Statistical Package for the Social Sciences (SPSS, Version 26.0, IBM, Armonk, NY, USA). Independent factorial ANOVA was used in order to 

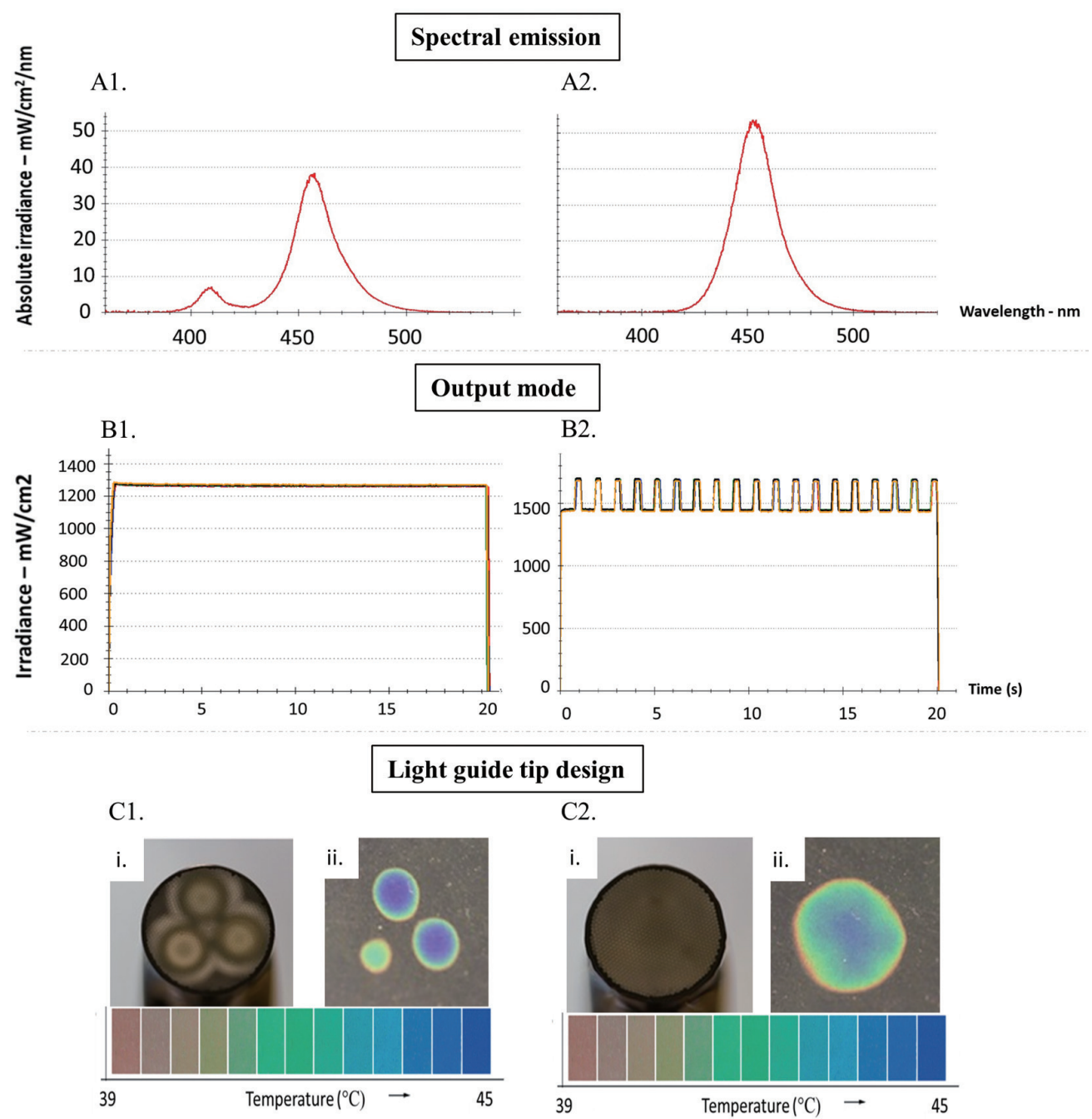

Fig. 1 Illustration of the different characteristics of the LED-LCUs investigated.

A: spectral emission with A1. Emission spectrum at $0 \mathrm{~mm}$ from Bluephase $\mathrm{G} 2^{\circledR}$ high mode illustrating wide-spectrum LED-LCUs and A2. from Elipar ${ }^{\mathrm{tm}}$ S10 illustrating narrow-spectrum LED-LCUs. B: Output mode with B1. Bluephase G2 ${ }^{\circledR}$ high mode illustrating continuous output mode LED-LCUs and B2. Demi ${ }^{\text {tm }}$ Ultra illustrating modulated output mode LED-LCUs. C: Light guide tip design. Heat distribution pattern of the LED-LCUs light guide tip in direct contact with a liquid crystal sheet. i. Photograph of LED-LCU light guide tips. ii. Photograph of liquid crystal sheet in direct contact with LED-LCU light guide tips. C1. Bluephase Style ${ }^{\circledR}$ (battery powered) light guide tip of older generation illustrating LED-LCUs delivering inhomogeneous beam. C2. Bluephase Style ${ }^{\circledR}$ (mains powered) light guide tip of newer generation illustrating LED-LCUs delivering homogeneous beam.

compare temperature increase in the pulp chamber and on the surface of the tooth between narrow-spectrum $v s$. wide-spectrum LED-LCUs, modulated output mode $v s$. continuous output mode, and light guide tip homogeneous vs. light guide tip inhomogeneous. Multivariable linear regression analysis was used in order to investigate the adjusted effect of factors influencing temperature development. Two models were constructed for each dependent variable stratified by spectral emission profile as there are indications that the pattern of the temperature development is different between wide- and narrow-spectrum LED-LCUs. Dependent variables were temperature increase on the surface and temperature increase in the pulp chamber; independent variables in model 1 were time, irradiance, distance, output mode and light guide tip design, while in model 2 independent variables were radiant exposure, distance, output mode and light guide tip design. Output mode variable was absent from the wide-spectrum LED-LCUs strata, and light guide tip design with inhomogeneous beam was absent from the narrow-spectrum LED-LCUs strata, since there were no such LED-LCUs in our sample. The 
Table 2 Radiant exposure (product of irradiance and time - Joule per $\mathrm{cm}^{2}$ ) delivered by each LED-LCU at 0,2 and $4 \mathrm{~mm}$ distance with $10 \mathrm{~s}$ and $20 \mathrm{~s}$ curing time

\begin{tabular}{|c|c|c|c|}
\hline $\begin{array}{l}\text { LED Light curing unit } \\
\text { (spectral emission, output mode, light guide tip design) }\end{array}$ & $\begin{array}{l}\text { Distance } \\
(\mathrm{mm})\end{array}$ & Time (s) & $\begin{array}{l}\text { Radiant exposure } \\
\left(\mathrm{J} / \mathrm{cm}^{2}\right)\end{array}$ \\
\hline $\begin{array}{l}\text { Bluephase Style }{ }^{\circledR} \text { battery } \\
\text { (wide-spectrum, continuous, inhomogeneous beam) }\end{array}$ & $\begin{array}{l}0 \\
2 \\
4\end{array}$ & $\begin{array}{l}10 \\
20 \\
10 \\
20 \\
10 \\
20\end{array}$ & $\begin{array}{l}12.2 \\
24.4 \\
15.3 \\
30.7 \\
13.7 \\
27.3\end{array}$ \\
\hline $\begin{array}{l}\text { Bluephase Style }{ }^{\circledR} \text { mains } \\
\text { (wide-spectrum, continuous, homogeneous beam) }\end{array}$ & $\begin{array}{l}0 \\
2 \\
4\end{array}$ & $\begin{array}{l}10 \\
20 \\
10 \\
20 \\
10 \\
20\end{array}$ & $\begin{array}{l}12.6 \\
25.2 \\
12.5 \\
25 \\
11.8 \\
23.5\end{array}$ \\
\hline $\begin{array}{l}\text { Bluephase G } 2^{\circledR} \text { High mode } \\
\text { (wide-spectrum, continuous, homogeneous beam) }\end{array}$ & $\begin{array}{l}0 \\
2 \\
4\end{array}$ & $\begin{array}{l}10 \\
20 \\
10 \\
20 \\
10 \\
20\end{array}$ & $\begin{array}{l}14.4 \\
28.7 \\
14.1 \\
28.3 \\
15.1 \\
30.2\end{array}$ \\
\hline $\begin{array}{l}\text { Bluephase G } 2^{\circledR} \text { Low mode } \\
\text { (wide-spectrum, continuous, homogeneous beam) }\end{array}$ & $\begin{array}{l}0 \\
2 \\
4\end{array}$ & $\begin{array}{l}10 \\
20 \\
10 \\
20 \\
10 \\
20\end{array}$ & $\begin{array}{r}7.7 \\
15.5 \\
8.3 \\
16.6 \\
8.6 \\
17.3\end{array}$ \\
\hline $\begin{array}{l}\text { TransLux }{ }^{\circledR} 2 \text { Wave } \\
\text { (wide-spectrum, continuous, homogeneous beam) }\end{array}$ & $\begin{array}{l}0 \\
2 \\
4\end{array}$ & $\begin{array}{l}10 \\
20 \\
10 \\
20 \\
10 \\
20\end{array}$ & $\begin{array}{l}13.6 \\
27.2 \\
13.4 \\
26.8 \\
13 \\
26\end{array}$ \\
\hline $\begin{array}{l}\text { TransLux }{ }^{\circledR} \text { Wave } \\
\text { (narrow-spectrum, continuous, homogeneous beam) }\end{array}$ & $\begin{array}{l}0 \\
2 \\
4\end{array}$ & $\begin{array}{l}10 \\
20 \\
10 \\
20 \\
10 \\
20\end{array}$ & $\begin{array}{l}16.7 \\
33.5 \\
16.9 \\
33.9 \\
15.4 \\
30.9\end{array}$ \\
\hline $\begin{array}{l}\text { Elipar }^{\mathrm{tm}} \text { DeepCure } \\
\text { (narrow-spectrum, continuous, homogeneous beam) }\end{array}$ & $\begin{array}{l}0 \\
2 \\
4\end{array}$ & $\begin{array}{l}10 \\
20 \\
10 \\
20 \\
10 \\
20\end{array}$ & $\begin{array}{l}23.7 \\
47.3 \\
20.4 \\
40.9 \\
17.8 \\
35.6\end{array}$ \\
\hline $\begin{array}{l}\text { Elipartm } \mathrm{S} 10 \\
\text { (narrow-spectrum, continuous, homogeneous beam) }\end{array}$ & $\begin{array}{l}0 \\
2 \\
4\end{array}$ & $\begin{array}{l}10 \\
20 \\
10 \\
20 \\
10 \\
20\end{array}$ & $\begin{array}{l}20.4 \\
40.9 \\
19.4 \\
38.7 \\
15.1 \\
30.2\end{array}$ \\
\hline $\begin{array}{l}\text { SmartLite }{ }^{\circledR} \text { Focus } \\
\text { (narrow-spectrum, continuous, homogeneous beam) }\end{array}$ & $\begin{array}{l}0 \\
2 \\
4\end{array}$ & $\begin{array}{l}10 \\
20 \\
10 \\
20 \\
10 \\
20\end{array}$ & $\begin{array}{l}10.3 \\
20.6 \\
11.9 \\
23.8 \\
10.2 \\
20.3\end{array}$ \\
\hline $\begin{array}{l}\text { Demi }{ }^{\text {tm }} \text { Ultra } \\
\text { (narrow-spectrum, modulated, homogeneous beam) }\end{array}$ & $\begin{array}{l}0 \\
2 \\
4\end{array}$ & $\begin{array}{l}10 \\
20 \\
10 \\
20 \\
10 \\
20\end{array}$ & $\begin{array}{c}14.9 \\
29.8 \\
13.9 \\
27.9 \\
9 \\
18\end{array}$ \\
\hline
\end{tabular}




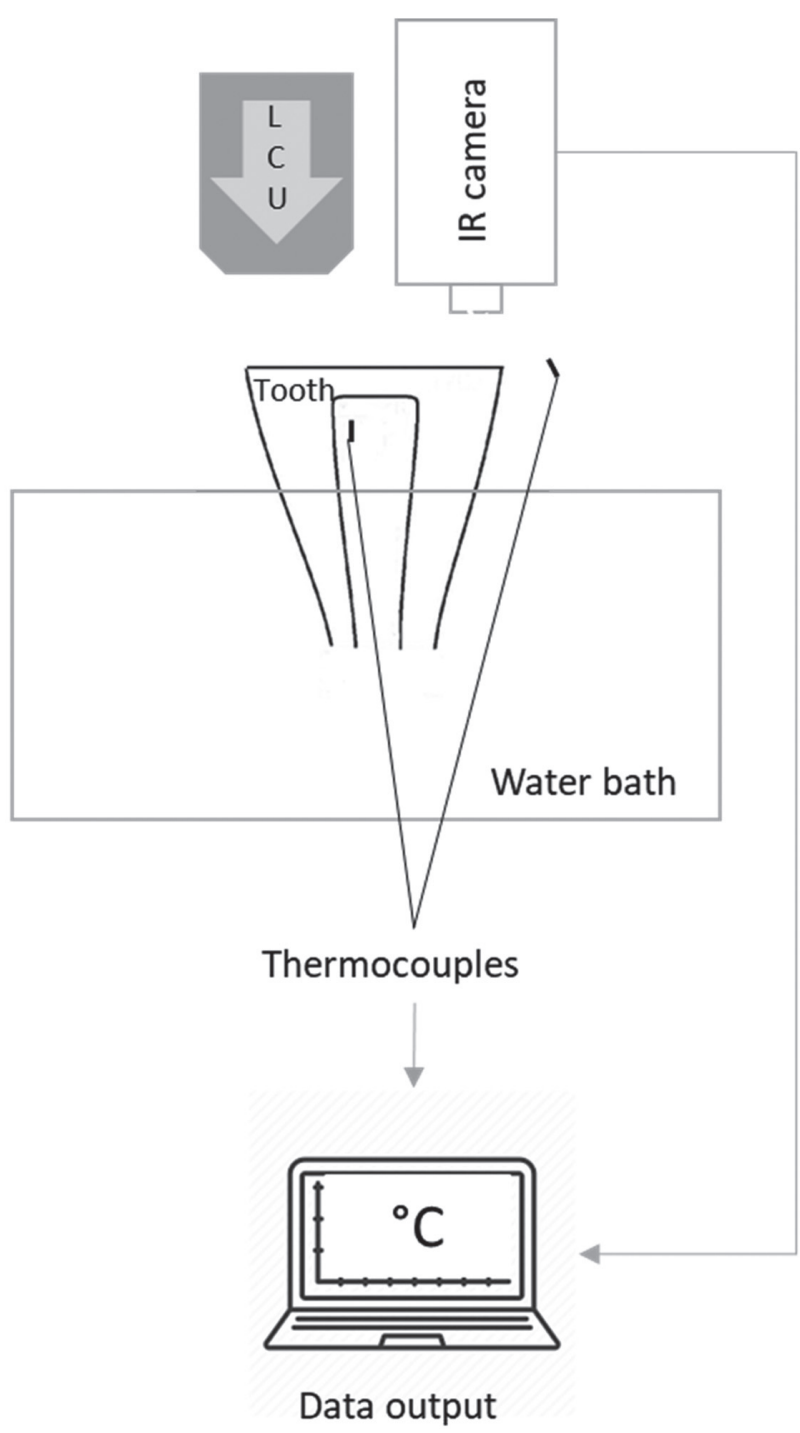

Fig. 2 Experimental setup for the measurement of the temperature development with thermocouples for the pulp chamber and infrared camera (IR camera) for the surface of a tooth when using different LED-LCU.

importance of predictor variables in linear regression models was determined by standardized regression coefficient. The statistical significance was set at $p<0.05$ and odds ratios are presented with $95 \%$ confidence intervals (CI).

\section{RESULTS}

\section{Temperature development}

The highest mean temperature increase in the pulp chamber was $6.1 \pm 0.3^{\circ} \mathrm{C}$ with a maximum temperature of $41.9^{\circ} \mathrm{C}$ at $2 \mathrm{~mm}$ distance and $20 \mathrm{~s} \mathrm{CT}$ with Bluephase G2 ${ }^{\circledR}$ high mode (featuring wide-spectrum spectral emission, continuous output mode and light guide tip homogeneous), while the highest mean temperature increase on the tooth surface was $20.1 \pm 1.7^{\circ} \mathrm{C}$ with a maximum temperature of $54.1^{\circ} \mathrm{C}$ at $2 \mathrm{~mm}$ distance and $20 \mathrm{~s}$ CT with Elipar ${ }^{\mathrm{tm}}$ DeepCure (featuring narrowspectrum spectral emission, continuous output mode and light guide tip homogeneous) (Table 3). The lowest mean temperature increase in the pulp chamber was $1.0 \pm 0.1^{\circ} \mathrm{C}$ with a maximum temperature of $36.8^{\circ} \mathrm{C}$ at 4 mm distance and $10 \mathrm{~s} \mathrm{CT}$ with Bluephase $\mathrm{G} 2^{\circledR}$ low mode (featuring wide-spectrum spectral emission, continuous output mode and light guide tip homogeneous) and $4.7 \pm 0.4^{\circ} \mathrm{C}$ with a maximum of $39^{\circ} \mathrm{C}$ at $4 \mathrm{~mm}$ distance and $10 \mathrm{~s}$ CT with SmartLite ${ }^{\circledR}$ Focus (featuring narrowspectrum spectral emission, continuous output mode and light guide tip homogeneous) for the surface temperature (Table 3).

\section{Effect of spectral emission}

Wide-spectrum LED-LCUs (vs. narrow-spectrum LEDLCUs) had a statistically significant higher temperature increase in the pulp chamber at $0 \mathrm{~mm}$ and $2 \mathrm{~mm}$ distance, and at $20 \mathrm{~s} \mathrm{CT}$, while narrow-spectrum LED-LCUs had a higher temperature increase at $4 \mathrm{~mm}$ distance (the significance was marginal, $p=0.05$ ) (Table 4).

Concerning the surface temperature, narrowspectrum LED-LCUs (vs. wide-spectrum LED-LCUs) produced statistically significantly higher temperature increase at $0 \mathrm{~mm}$ and $2 \mathrm{~mm}$ distances, and $10 \mathrm{~s}$ and $20 \mathrm{~s}$ CT (Table 4).

\section{Effect of output mode}

Modulated output mode LED-LCUs (featuring narrowspectrum spectral emission) resulted in a statistically significantly lower temperature increase in the pulp chamber compared to continuous output mode for $10 \mathrm{~s}$ and $20 \mathrm{~s} \mathrm{CT}$, and $0 \mathrm{~mm}$ and $2 \mathrm{~mm}$ distance. On the tooth surface, modulated output mode (vs. continuous) had a higher temperature increase at $0 \mathrm{~mm}$ distance and at $10 \mathrm{~s}$ and $20 \mathrm{~s} \mathrm{CT}$ (Table 4). According to multivariable linear regression, modulated output mode $v s$. continuous increased surface temperature by $4.9^{\circ} \mathrm{C}\left(95 \% \mathrm{CI} 4.2^{\circ} \mathrm{C}\right.$ to $5.7^{\circ} \mathrm{C}$ ) and was the second strongest predictor after irradiance (Table 5). Moreover, modulated output mode versus continuous output mode resulted in $1.0^{\circ} \mathrm{C}(95 \%$ $\mathrm{CI}-1.3^{\circ} \mathrm{C}$ to $-0.7^{\circ} \mathrm{C}$ ) lower pulp chamber temperature increase (Table 5).

\section{Effect of light guide tip design}

The heat distribution pattern showed that the heat was inhomogeneously distributed across the tip of Bluephase Style ${ }^{\circledR}$ battery that had older light guide tip design (Fig. 1, C1ii.). For other LED-LCUs' light guide tips the heat distribution was more homogenous, with the highest temperature being recorded at the center of the tip surface, decreasing towards the periphery of the measured temperature field. LED-LCU with inhomogeneous light guide tip (featuring wide-spectrum spectral emission) resulted in greater increases in surface temperature compared to LED-LCU with homogeneous light guide tips at all $\mathrm{CT}$ and distances tested, and also greater increases in pulp chamber temperature at $10 \mathrm{~s}$ 
Table 3 Mean temperature increases in the pulp chamber (Chamber) and at the surface of the tooth (Surface) (in ${ }^{\circ} \mathrm{C}$ ) with standard deviation (SD) following exposure with different LED-LCUs.

\begin{tabular}{|c|c|c|c|c|c|c|c|c|c|c|c|c|}
\hline \multirow{2}{*}{$\begin{array}{l}\text { LED-Light curing } \\
\text { unit (spectral } \\
\text { emission, output } \\
\text { mode, light guide } \\
\text { tip design) }\end{array}$} & \multicolumn{2}{|c|}{$0 \mathrm{~mm} 10 \mathrm{~s}$} & \multicolumn{2}{|c|}{$0 \mathrm{~mm} 20 \mathrm{~s}$} & \multicolumn{2}{|c|}{$2 \mathrm{~mm} 10 \mathrm{~s}$} & \multicolumn{2}{|c|}{$2 \mathrm{~mm} 20 \mathrm{~s}$} & \multicolumn{2}{|c|}{$4 \mathrm{~mm} 10 \mathrm{~s}$} & \multicolumn{2}{|c|}{$4 \mathrm{~mm} 20 \mathrm{~s}$} \\
\hline & Chamber & Surface & Chamber & Surface & Chamber & Surface & Chamber & Surface & Chamber & Surface & Chamber & Surface \\
\hline $\begin{array}{l}\text { Bluephase } \\
\text { Style }^{\circledR} \text { battery } \\
\text { (wide-spectrum, } \text { continuous, } \\
\text { inhomogeneous } \\
\text { beam) }\end{array}$ & $\begin{array}{c}4.0 \\
(0.2)\end{array}$ & $\begin{array}{c}15 \\
(0.7)\end{array}$ & $\begin{array}{c}5.6 \\
(0.3)\end{array}$ & $\begin{array}{l}19.0 \\
(0.4)\end{array}$ & $\begin{array}{c}3.1 \\
(0.3)\end{array}$ & $\begin{array}{l}14.8 \\
(0.7)\end{array}$ & $\begin{array}{c}5.4 \\
(0.4)\end{array}$ & $\begin{array}{l}18.4 \\
(0.5)\end{array}$ & $\begin{array}{c}1.8 \\
(0.2)\end{array}$ & $\begin{array}{l}12.9 \\
(1.3)\end{array}$ & $\begin{array}{c}3.4 \\
(0.5)\end{array}$ & $\begin{array}{l}17.0 \\
(0.3)\end{array}$ \\
\hline $\begin{array}{l}\text { Bluephase Style } \\
\text { mains } \\
\text { (wide-spectrum, } \\
\text { continuous, } \\
\text { homogeneous } \\
\text { beam) }\end{array}$ & $\begin{array}{c}2.5 \\
(0.1)\end{array}$ & $\begin{array}{c}7.3 \\
(0.4)\end{array}$ & $\begin{array}{c}4.4 \\
(0.2)\end{array}$ & $\begin{array}{l}10.1 \\
(0.4)\end{array}$ & $\begin{array}{c}2.0 \\
(0.2)\end{array}$ & $\begin{array}{c}7.8 \\
(0.2)\end{array}$ & $\begin{array}{c}3.8 \\
(0.2)\end{array}$ & $\begin{array}{l}10.8 \\
(0.3)\end{array}$ & $\begin{array}{c}1.5 \\
(0.1)\end{array}$ & $\begin{array}{c}6.4 \\
(0.5)\end{array}$ & $\begin{array}{c}2.5 \\
(0.2)\end{array}$ & $\begin{array}{c}8.7 \\
(0.4)\end{array}$ \\
\hline $\begin{array}{l}\text { Bluephase G2 }{ }^{\circledR} \\
\text { High Mode } \\
\text { (wide-spectrum, } \\
\text { continuous, } \\
\text { homogeneous } \\
\text { beam) }\end{array}$ & $\begin{array}{c}3.0 \\
(0.2)\end{array}$ & $\begin{array}{c}9.1 \\
(0.3)\end{array}$ & $\begin{array}{c}5.4 \\
(0.3)\end{array}$ & $\begin{array}{l}12.4 \\
(0.6)\end{array}$ & $\begin{array}{c}3.2 \\
(0.1)\end{array}$ & $\begin{array}{l}11.2 \\
(0.6)\end{array}$ & $\begin{array}{c}6.1 \\
(0.3)\end{array}$ & $\begin{array}{l}16.2 \\
(0.1)\end{array}$ & $\begin{array}{c}2.0 \\
(0.2)\end{array}$ & $\begin{array}{l}10.0 \\
(0.3)\end{array}$ & $\begin{array}{c}3.5 \\
(0.2)\end{array}$ & $\begin{array}{l}13.5 \\
(1.0)\end{array}$ \\
\hline $\begin{array}{l}\text { Bluephase } \mathrm{G} 2^{\circledR} \\
\text { Low Mode } \\
\text { (wide-spectrum, } \\
\text { continuous, } \\
\text { homogeneous } \\
\text { beam) }\end{array}$ & $\begin{array}{c}1.6 \\
(0.2)\end{array}$ & $\begin{array}{c}5.3 \\
(0.2)\end{array}$ & $\begin{array}{c}2.9 \\
(0.2)\end{array}$ & $\begin{array}{c}7.3 \\
(0.3)\end{array}$ & $\begin{array}{c}1.2 \\
(0.1)\end{array}$ & $\begin{array}{c}5.4 \\
(0.2)\end{array}$ & $\begin{array}{c}2.2 \\
(0.2)\end{array}$ & $\begin{array}{c}7.7 \\
(0.2)\end{array}$ & $\begin{array}{c}1.0 \\
(0.1)\end{array}$ & $\begin{array}{c}5.8 \\
(0.3)\end{array}$ & $\begin{array}{c}1.8 \\
(0.1)\end{array}$ & $\begin{array}{c}8.2 \\
(0.1)\end{array}$ \\
\hline $\begin{array}{l}\text { Translux }{ }^{\circledR} 2 \text { Wave } \\
\text { (wide-spectrum, } \\
\text { continuous, } \\
\text { homogeneous } \\
\text { beam) }\end{array}$ & $\begin{array}{c}3.1 \\
(0.1)\end{array}$ & $\begin{array}{c}7.4 \\
(0.3)\end{array}$ & $\begin{array}{c}4.9 \\
(0.2)\end{array}$ & $\begin{array}{l}10.4 \\
(0.3)\end{array}$ & $\begin{array}{c}3.0 \\
(0.1)\end{array}$ & $\begin{array}{c}8.2 \\
(0.3)\end{array}$ & $\begin{array}{c}5.2 \\
(0.2)\end{array}$ & $\begin{array}{l}11.2 \\
(0.3)\end{array}$ & $\begin{array}{c}3.0 \\
(0.1)\end{array}$ & $\begin{array}{c}7.3 \\
(0.1)\end{array}$ & $\begin{array}{c}4.8 \\
(0.1)\end{array}$ & $\begin{array}{c}9.9 \\
(0.4)\end{array}$ \\
\hline $\begin{array}{l}\text { Translux }{ }^{\circledR} \text { Wave } \\
\text { (narrow-spectrum, } \\
\text { continuous, } \\
\text { homogeneous } \\
\text { beam) }\end{array}$ & $\begin{array}{c}3.0 \\
(0.3)\end{array}$ & $\begin{array}{c}9.8 \\
(0.6)\end{array}$ & $\begin{array}{c}4.4 \\
(0.7)\end{array}$ & $\begin{array}{l}13.5 \\
(0.9)\end{array}$ & $\begin{array}{c}2.7 \\
(0.2)\end{array}$ & $\begin{array}{l}10.7 \\
(0.8)\end{array}$ & $\begin{array}{c}3.3 \\
(0.2)\end{array}$ & $\begin{array}{l}15.2 \\
(0.3)\end{array}$ & $\begin{array}{c}2.3 \\
(0.2)\end{array}$ & $\begin{array}{c}9.5 \\
(0.4)\end{array}$ & $\begin{array}{c}2.6 \\
(0.1)\end{array}$ & $\begin{array}{l}14.6 \\
(1.0)\end{array}$ \\
\hline $\begin{array}{l}\text { Elipar }{ }^{\mathrm{tm}} \text { DeepCure } \\
\text { (narrow-spectrum, } \\
\text { continuous, } \\
\text { homogeneous } \\
\text { beam) }\end{array}$ & $\begin{array}{c}3.5 \\
(0.4)\end{array}$ & $\begin{array}{l}12.4 \\
(0.6)\end{array}$ & $\begin{array}{c}4.4 \\
(0.2)\end{array}$ & $\begin{array}{l}17.5 \\
(1.0)\end{array}$ & $\begin{array}{c}2.5 \\
(0.0)\end{array}$ & $\begin{array}{l}15.8 \\
(1.0)\end{array}$ & $\begin{array}{c}5.3 \\
(0.5)\end{array}$ & $\begin{array}{l}20.1 \\
(1.7)\end{array}$ & $\begin{array}{c}2.7 \\
(0.1)\end{array}$ & $\begin{array}{l}13.7 \\
(0.4)\end{array}$ & $\begin{array}{c}5.3 \\
(0.3)\end{array}$ & $\begin{array}{l}17.1 \\
(1.1)\end{array}$ \\
\hline $\begin{array}{l}\text { Elipar }^{\text {tm }} \text { S10 } \\
\text { (narrow-spectrum, } \\
\text { continuous, } \\
\text { homogeneous } \\
\text { beam) }\end{array}$ & $\begin{array}{c}3.2 \\
(0.2)\end{array}$ & $\begin{array}{l}13.1 \\
(0.9)\end{array}$ & $\begin{array}{c}3.4 \\
(0.2)\end{array}$ & $\begin{array}{l}16.1 \\
(0.8)\end{array}$ & $\begin{array}{c}2.7 \\
(0.2)\end{array}$ & $\begin{array}{l}11.5 \\
(0.6)\end{array}$ & $\begin{array}{c}4.0 \\
(0.2)\end{array}$ & $\begin{array}{l}14.9 \\
(1.0)\end{array}$ & $\begin{array}{c}3.0 \\
(0.1)\end{array}$ & $\begin{array}{l}10.4 \\
(0.5)\end{array}$ & $\begin{array}{c}3.5 \\
(0.3)\end{array}$ & $\begin{array}{l}15.1 \\
(0.7)\end{array}$ \\
\hline $\begin{array}{l}\text { SmartLite }{ }^{\circledR} \text { Focus } \\
\text { (narrow-spectrum, } \\
\text { continuous, } \\
\text { homogeneous } \\
\text { beam) }\end{array}$ & $\begin{array}{c}2.3 \\
(0.2)\end{array}$ & $\begin{array}{c}6.9 \\
(0.5)\end{array}$ & $\begin{array}{c}3.7 \\
(0.5)\end{array}$ & $\begin{array}{c}7.1 \\
(0.2)\end{array}$ & $\begin{array}{c}2.7 \\
(0.2)\end{array}$ & $\begin{array}{c}5.3 \\
(0.3)\end{array}$ & $\begin{array}{c}3.4 \\
(0.2)\end{array}$ & $\begin{array}{c}7.7 \\
(0.7)\end{array}$ & $\begin{array}{c}1.8 \\
(0.1)\end{array}$ & $\begin{array}{c}4.7 \\
(0.4)\end{array}$ & $\begin{array}{c}2.7 \\
(0.2)\end{array}$ & $\begin{array}{c}5.6 \\
(0.3)\end{array}$ \\
\hline $\begin{array}{l}\text { Demi }{ }^{\text {tm }} \text { Ultra } \\
\text { (narrow-spectrum, } \\
\text { modulated, } \\
\text { homogeneous } \\
\text { beam) }\end{array}$ & $\begin{array}{c}0.9 \\
(0.1)\end{array}$ & $\begin{array}{l}13.7 \\
(0.8)\end{array}$ & $\begin{array}{c}1.8 \\
(0.2)\end{array}$ & $\begin{array}{l}18.5 \\
(1.1)\end{array}$ & $\begin{array}{c}1.1 \\
(0.1)\end{array}$ & $\begin{array}{l}12.2 \\
(0.6)\end{array}$ & $\begin{array}{c}3.5 \\
(0.3)\end{array}$ & $\begin{array}{l}15.7 \\
(0.9)\end{array}$ & $\begin{array}{c}1.5 \\
(0.1)\end{array}$ & $\begin{array}{c}8.7 \\
(0.5)\end{array}$ & $\begin{array}{c}2.9 \\
(0.4)\end{array}$ & $\begin{array}{l}11.6 \\
(0.5)\end{array}$ \\
\hline
\end{tabular}


Table 4 Mean temperature increase in the pulp chamber and at the surface of the tooth (in ${ }^{\circ} \mathrm{C}$ ) with standard deviation $(\mathrm{SD})$ following exposure with narrow-spectrum versus wide-spectrum; continuous output mode versus modulated output and light guide tip homogeneous versus light guide tip inhomogeneous LED-LCUs

\begin{tabular}{|c|c|c|c|c|c|c|c|c|}
\hline & & & $\begin{array}{l}\text { Narrow- } \\
\text { spectrum }^{1}\end{array}$ & $\begin{array}{l}\text { Wide- } \\
\text { spectrum }^{2}\end{array}$ & Continuous $^{3}$ & Modulated $^{4}$ & Homogeneous $^{5}$ & Inhomogeneous $^{6}$ \\
\hline \multirow{5}{*}{$\begin{array}{l}\text { Pulp chamber } \\
\text { temperature }\end{array}$} & \multirow{3}{*}{ Distance } & $0 \mathrm{~mm}$ & $3.1(1.1)^{*}$ & $3.7(1.3)^{*}$ & $3.6(1.1)^{*}$ & $1.4(0.5)^{*}$ & $3.2(1.2)^{*}$ & $4.8(0.8)^{*}$ \\
\hline & & $2 \mathrm{~mm}$ & $3.1(1.1)^{*}$ & $3.5(1.5)^{*}$ & $3.4(1.3)^{*}$ & $2.3(1.3)^{*}$ & $3.2(1.3)^{*}$ & $4.3(1.3)^{*}$ \\
\hline & & $4 \mathrm{~mm}$ & $2.8(1.0)$ & $2.5(1.3)$ & $2.7(1.2)$ & $2.2(0.8)$ & $2.7(1.2)$ & $2.6(0.9)$ \\
\hline & \multirow{2}{*}{ Time } & $10 \mathrm{~s}$ & $2.4(0.8)$ & $2.4(1.0)$ & $2.5(0.8)^{*}$ & $1.1(0.3)^{*}$ & $2.3(0.9)^{*}$ & $3.0(1.0)^{*}$ \\
\hline & & $20 \mathrm{~s}$ & $3.6(1.0)^{*}$ & $4.1(1.3)^{*}$ & $4.0(1.2)^{*}$ & $2.7(0.8)^{*}$ & $3.8(1.2)^{*}$ & $4.8(1.1)^{*}$ \\
\hline \multirow{5}{*}{$\begin{array}{l}\text { Surface } \\
\text { temperature }\end{array}$} & \multirow{3}{*}{ Distance } & $0 \mathrm{~mm}$ & $12.9(3.9)^{*}$ & $10.2(3.8)^{*}$ & $11.0(3.9)^{*}$ & $16.1(2.7)^{*}$ & $11.0(3.9)^{*}$ & $16.8(2.1)^{*}$ \\
\hline & & $2 \mathrm{~mm}$ & $12.9(4.2)^{*}$ & $11.2(4.0)^{*}$ & $11.8(4.3)$ & $14.0(2.0)$ & $11.5(4.1)^{*}$ & $16.6(2.0)^{*}$ \\
\hline & & $4 \mathrm{~mm}$ & $11.1(4.0)$ & $10.0(3.4)$ & $10.6(3.9)$ & $10.2(1.6)$ & $10.0(3.5)^{*}$ & $15.0(2.4)^{*}$ \\
\hline & \multirow{2}{*}{ Time } & $10 \mathrm{~s}$ & $10.6(3.1)^{*}$ & $8.9(3.2)^{*}$ & $9.5(3.3)^{*}$ & $11.5(2.3)^{*}$ & $9.2(3.0)^{*}$ & $14.2(1.3)^{*}$ \\
\hline & & $20 \mathrm{~s}$ & $14.0(4.2)^{*}$ & $12.0(3.7)^{*}$ & $12.7(4.1)^{*}$ & $15.3(3.0)^{*}$ & $12.5(4.0)^{*}$ & $18.1(0.9)^{*}$ \\
\hline
\end{tabular}

${ }^{1}$ Narrow-spectrum: TransLux ${ }^{\circledR}$ Wave; Elipar ${ }^{\text {tm }}$ DeepCure; Elipar ${ }^{\text {tm }}$ S10; SmartLite ${ }^{\circledR}$ Focus; Demi ${ }^{\text {tm }}$ Ultra

${ }^{2}$ Wide-spectrum: Bluephase Style ${ }^{\circledR}$ battery; Bluephase Style ${ }^{\circledR}$ mains; Bluephase G2 ${ }^{\circledR}$ High mode; Bluephase G2 ${ }^{\circledR}$ Low mode; TransLux $^{\circledR}$ 2Wave

${ }^{3}$ Continuous output mode: Bluephase Style ${ }^{\circledR}$ battery; Bluephase Style ${ }^{\circledR}$ mains; Bluephase G2 ${ }^{\circledR}$ High mode; Bluephase G2 ${ }^{\circledR}$ Low mode; TransLux ${ }^{\circledR}$ 2Wave; TransLux ${ }^{\circledR}$ Wave; Elipar ${ }^{\text {tm }}$ DeepCure; Elipar ${ }^{\text {tm }}$ S10; SmartLite ${ }^{\circledR}$ Focus

${ }^{4}$ Modulated output mode: Demi ${ }^{\text {tm }}$ Ultra

${ }^{5}$ Homogeneous light guide tip: Bluephase Style ${ }^{\circledR}$ mains; Bluephase G2 ${ }^{\circledR}$ High mode; Bluephase G2 ${ }^{\circledR}$ Low mode; TransLux ${ }^{\circledR}$ 2Wave; TransLux ${ }^{\circledR}$ Wave; Elipar ${ }^{\mathrm{tm}}$ DeepCure; Elipar ${ }^{\mathrm{tm}}$ S10; SmartLite $^{\circledR}$ Focus; Demi $^{\text {tm }}$ Ultra

${ }^{6}$ Inhomogeneous light guide tip: Bluephase Style ${ }^{\circledR}$ battery

*According to independent factorial ANOVA $p<0.05$ within distance and time separately under the same LED-LCU characteristic.

and $20 \mathrm{~s}$ CT and $0 \mathrm{~mm}$ and $2 \mathrm{~mm}$ distances (Table 4). According to multivariable linear regression, light guide tip delivering an inhomogeneous beam vs. homogeneous beam-resulted in a surface temperature increase of $5.9^{\circ} \mathrm{C}$ $\left(95 \% \mathrm{CI} 5.4^{\circ} \mathrm{C}\right.$ to $\left.6.5^{\circ} \mathrm{C}\right)$ and was the strongest predictor for surface temperature increase followed by irradiance (Table 5). For pulp chamber temperature increase both for narrow- and wide-spectrum LED-LCUs time was the strongest predictor.

\section{DISCUSSION}

To the best of our knowledge, this is the first study that compared the influence of LED-LCUs spectral emission, output mode and light guide tip design on the temperature development in the pulp chamber and on tooth surface. LED-LCUs with different spectral emission contributed to different temperature development. Wide-spectrum LED-LCUs produced higher temperature increase in the pulp chamber at distances of $0 \mathrm{~mm}$ as well as at $2 \mathrm{~mm}$ while narrow-spectrum LED-LCUs at $4 \mathrm{~mm}$ distance. For the surface temperature development, narrowspectrum LED-LCUs produced higher temperature increase. LED-LCU featuring modulated output mode (compared to LED-LCUs featuring continuous mode) resulted in lower increase in pulp chamber temperature, but higher increase on surface temperature. LEDLCU with light guide tip delivering an inhomogeneous beam (compared to LED-LCUs with light guide tips delivering a homogenous beam) caused higher increase in temperature on the surface and in the pulp chamber. Therefore, our null-hypothesis, stating that there were no differences in the temperature development (on the tooth surface and pulp chamber) when exposed to LEDLCUs having different spectral emission, output mode and light guide tip design, was rejected.

\section{Methodological considerations}

In the present study we used an in vitro model in teeth without blood supply which may have some effect on heat transfer. However, this may be of minor concern since the absolute amount of circulating blood in an intact tooth is very small ${ }^{30)}$. Moreover, it has been shown that in clinical situation where local anesthetics with vasoconstrictors are used, a marked, sustained decrease of blood flow in the pulp chamber was observed ${ }^{31}$. Our experimental setup can therefore be considered as a worst case clinical situation, taking additionally into consideration the small thickness of remaining dentin. A study comparing in vitro and in vivo models to investigate temperature increase when exposed to LED-LCUs concluded that at a clinical relevant CT (such as $20 \mathrm{~s}$ in our study) only small differences of temperature development were observed between the two models ${ }^{32}$. In addition, in vitro model can be considered advantageous over in vivo model for ethical reasons. Of note, the positioning of the probe 
Table 5 The adjusted effect of curing time (CT), output mode, irradiance, distance, light guide tip design and radiant exposure (product of irradiance and time) on temperature of the surface and pulp chamber in a tooth according to multivariable linear regression analyses

\begin{tabular}{|c|c|c|c|c|c|}
\hline & & \multicolumn{2}{|c|}{ Narrow-spectrum LED-LCU } & \multicolumn{2}{|c|}{ Wide-spectrum LED-LCU } \\
\hline & & $\begin{array}{l}\text { Surface } \\
\text { temperature }\end{array}$ & $\begin{array}{l}\text { Pulp chamber } \\
\text { temperature }\end{array}$ & $\begin{array}{l}\text { Surface } \\
\text { temperature }\end{array}$ & $\begin{array}{l}\text { Pulp chamber } \\
\text { temperature }\end{array}$ \\
\hline & $\begin{array}{l}\text { Independent } \\
\text { variable }\end{array}$ & \multicolumn{4}{|c|}{$\begin{array}{c}\mathrm{B}(95 \% \mathrm{CI}) \\
p\end{array}$} \\
\hline \multirow{6}{*}{$\begin{array}{l}\text { Model } \\
1\end{array}$} & $\begin{array}{l}\mathrm{CT} \\
20 \mathrm{~s} \\
(\text { vs. } 10 \mathrm{~s})\end{array}$ & $\begin{array}{l}3.5(2.9 \text { to } 4.0) \\
0.000\end{array}$ & $\begin{array}{l}1.2(1.0 \text { to } 1.4) \\
0.000\end{array}$ & $\begin{array}{l}3.1(2.7 \text { to } 3.5) \\
0.000\end{array}$ & $\begin{array}{l}1.8(1.5 \text { to } 2.0) \\
0.000\end{array}$ \\
\hline & $\begin{array}{l}\text { Output mode } \\
\text { Modulated } \\
\text { (vs. continuous) }\end{array}$ & $\begin{array}{l}4.9(4.2 \text { to } 5.7) \\
0.000\end{array}$ & $\begin{array}{l}-1.0(-1.3 \text { to }-0.7) \\
0.000\end{array}$ & - & - \\
\hline & $\begin{array}{l}\text { Irradiance } \\
\text { (per } 100 \text { units) }\end{array}$ & $\begin{array}{l}0.9(0.8 \text { to } 1.0) \\
0.000\end{array}$ & $\begin{array}{l}0.1(0.1 \text { to } 0.1) \\
0.000\end{array}$ & $\begin{array}{l}0.7(0.6 \text { to } 0.8) \\
0.000\end{array}$ & $\begin{array}{l}0.3(0.3 \text { to } 0.4) \\
0.000\end{array}$ \\
\hline & $\begin{array}{l}\text { Distance } \\
2 \mathrm{~mm} \\
(v s .0 \mathrm{~mm}) \\
4 \mathrm{~mm} \\
(\text { vs. } 0 \mathrm{~mm})\end{array}$ & $\begin{array}{l}0.6(-0.4 \text { to } 1.4) \\
\mathrm{NS} \\
1.6(0.8 \text { to } 2.3) \\
0.000\end{array}$ & $\begin{array}{l}0.1(-0.1 \text { to } 0.4) \\
\text { NS } \\
0.1(-0.2 \text { to } 0.3) \\
\text { NS }\end{array}$ & $\begin{array}{l}0.5(-0.1 \text { to } 1.0) \\
\mathrm{NS} \\
-0.5(-1.1 \text { to }-0.03) \\
0.037\end{array}$ & $\begin{array}{l}-0.4(-0.7 \text { to }-0.2) \\
0.003 \\
-1.4(-1.7 \text { to }-1.1) \\
0.000\end{array}$ \\
\hline & $\begin{array}{l}\text { Light guide tip } \\
\text { inhomogeneous } \\
\text { (vs. homogeneous) }\end{array}$ & - & - & $\begin{array}{l}5.9(5.4 \text { to } 6.5) \\
0.000\end{array}$ & $\begin{array}{l}0.3(-0.01 \text { to } 0.6) \\
\text { NS }\end{array}$ \\
\hline & $\mathrm{R}^{2}$ & 0.82 & 0.66 & 0.89 & 0.78 \\
\hline \multirow{5}{*}{$\begin{array}{l}\text { Model } \\
2\end{array}$} & $\begin{array}{l}\text { Radiant exposure } \\
\text { (per } 10 \text { units) }\end{array}$ & $\begin{array}{l}0.3(0.3 \text { to } 0.4) \\
0.000\end{array}$ & $\begin{array}{l}0.1(0.05 \text { to } 0.1) \\
0.000\end{array}$ & $\begin{array}{l}2.9(2.6 \text { to } 3.2) \\
0.000\end{array}$ & $\begin{array}{l}1.6(1.4 \text { to } 1.7) \\
0.000\end{array}$ \\
\hline & $\begin{array}{l}\text { Output mode } \\
\text { (modulated } v s . \\
\text { continuous) }\end{array}$ & $\begin{array}{l}3.3(2.3 \text { to } 4.3) \\
0.000\end{array}$ & $\begin{array}{l}-0.9(-1.2 \text { to } 0.7) \\
0.000\end{array}$ & - & - \\
\hline & $\begin{array}{l}\text { Distance } \\
2 \mathrm{~mm} \\
\text { (vs. } 0 \mathrm{~mm}) \\
4 \mathrm{~mm} \\
\text { (vs. } 0 \mathrm{~mm})\end{array}$ & $\begin{array}{l}0.4(-0.6 \text { to } 1.3) \\
\text { NS } \\
0.05(-0.9 \text { to } 1.0) \\
\text { NS }\end{array}$ & $\begin{array}{l}0.1(-0.1 \text { to } 0.4) \\
\mathrm{NS} \\
0.2(-0.1 \text { to } 0.4) \\
\mathrm{NS}\end{array}$ & $\begin{array}{l}0.6(0.04 \text { to } 1.1) \\
0.034 \\
-0.5(-1.0 \text { to } 0.07) \\
\mathrm{NS}\end{array}$ & $\begin{array}{l}-0.4(-0.7 \text { to }-0.1) \\
0.008 \\
-1.3(-1.6 \text { to }-1.1) \\
0.000\end{array}$ \\
\hline & $\begin{array}{l}\text { Light guide tip } \\
\text { inhomogeneous } \\
\text { (vs. homogeneous) }\end{array}$ & - & - & $\begin{array}{l}6.3(5.8 \text { to } 6.9) \\
0.000\end{array}$ & $\begin{array}{l}0.4(0.1 \text { to } 0.7) \\
0.005\end{array}$ \\
\hline & $\mathrm{R}^{2}$ & 0.67 & 0.64 & 0.87 & 0.78 \\
\hline
\end{tabular}

NS: no statistical significance

into the pulp chamber might have underestimated the temperature development since it might have not been placed exactly where the highest temperature increase occurred. Temperature measurements with CT longer than $20 \mathrm{~s}$ were not tested in this study since this is not recommended in most LED-LCUs user manuals and six of the LED-LCUs had no such option. Nevertheless, a recent questionnaire study among Norwegian dentists showed that the average CT of restorations was around $30 \mathrm{~s}$ with a range extending to as much as $60 \mathrm{~s}^{3)}$. Hence, the temperature increase within the pulp chamber and at the surface of teeth is likely to be greater with extended $\mathrm{CT}^{8)}$. This is of particular importance since CT was the strongest predictor for pulp chamber temperature increase both for narrow- and wide-spectrum LEDLCUs.

In our study only one LED-LCU with modulated output mode was tested (Demi ${ }^{\text {tm }}$ Ultra with narrowspectrum) and it is not certain if other LED-LCUs having modulated output mode would behave in similar 
manner. Having only one LED-LCU in this study with modulated output mode is a reflection of the situation on the market where only few LED-LCUs with modulated output mode are available ${ }^{33}$. Similarly, there was only one LED-LCU that delivered an inhomogeneous beam in our sample. Considering these inherent limitations, the results for the output mode and light guide tip design of the LED-LCUs should be interpreted with caution.

\section{Temperature development}

The highest pulp chamber increase observed was $6.1 \pm 0.3^{\circ} \mathrm{C}$. In 1965 , in an in vivo study, performed in monkeys by applying a soldering iron on the tested teeth, Zach and Cohen showed that a sustained increase of $5.5^{\circ} \mathrm{C}$ in the pulp chamber was the threshold to determine whether there was a risk for necrosis of the pulp $^{11)}$. In 2015, Runnacles and co-workers performed an in vivo study on 14 years old patients' intact premolars. They demonstrated that wide-spectrum LED-LCU increased temperature in the pulp chamber with some cases exceeding the threshold of $5.5^{\circ} \mathrm{C}^{10)}$. A study using human teeth showed that a temporary temperature increase of $8.9^{\circ} \mathrm{C}$ to $14.7^{\circ} \mathrm{C}$ did not result in pulp necrosis. The authors explained differences in histological findings due to the higher rate of pulp chamber temperature increase in the methodology used by Zach and Cohen compared to their study ${ }^{34)}$. On the other hand, an earlier study reported that an increase of $11^{\circ} \mathrm{C}$ to $20^{\circ} \mathrm{C}$ in a vital tooth pulp for just $30 \mathrm{~s}$ may be harmful and cause irreversible changes in a pulp ${ }^{35}$. This would indicate, as suggested by others, that an amount of time above a threshold temperature would provide a more accurate description for potential thermal damage ${ }^{14)}$. One of the LED-LCUs in the present study exceeded the threshold of $5.5^{\circ} \mathrm{C}$; the temperature increase in the pulp chamber was $6.1^{\circ} \mathrm{C}$ reaching $41.9^{\circ} \mathrm{C}$ at $20 \mathrm{~s}$ exposure time.

Even though concerns have been raised regarding LED-LCUs' effect on temperature increase on oral soft tissues, little information on the subject is available in the literature ${ }^{8,36}$. An in vivo study on pigs showed that wide-spectrum LED-LCU placed at a distance of $5 \mathrm{~mm}$ from the gingival tissue increased the gingival temperature to $41^{\circ} \mathrm{C}$ and caused gingival lesion for $67 \%$ and $77 \%$ of the tissue at 40 and $60 \mathrm{~s} \mathrm{CT}$, respectively ${ }^{13)}$. The maximum temperature increase on the tooth surface observed in our study was $20.1^{\circ} \mathrm{C}$, reaching $54.1^{\circ} \mathrm{C}$, which might exceed critical temperature for soft tissue in connection to some clinical situations such as class $\mathrm{V}$ restorations.

\section{Effect of spectral emission}

In the present study, wide-spectrum LED-LCUs had a higher temperature increase in the pulp chamber compared to narrow-spectrum LED-LCUs at distance of $0 \mathrm{~mm}$ and $2 \mathrm{~mm}$.

In a recent study that used one of the wide-spectrum LED-LCU also tested in our work (Bluephase G2 ${ }^{\circledR}$, Ivoclar Vivadent), it has been shown that at $0 \mathrm{~mm}$ the violet light component represented $15 \%$ of the total light output. It has been reported that violet light (below 420 $\mathrm{nm}$ ) produces more energy compared to blue light (above $420 \mathrm{~nm}$ ); photons at $410 \mathrm{~nm}$ have been shown to deliver $12 \%$ more energy than photons at $460 \mathrm{~nm}$. Consequently $12 \%$ fewer photons are required to deliver the same amount of energy ${ }^{18}$. On the other hand, at a given radiant emittance, the higher temperature increase produced by wide-spectrum LED-LCU might be due to different absorption coefficients of the "blue" and "violet" lights rather than energy delivered by violet and blue lights $^{19,37-39}$. Clinically, during restorative procedure a tooth is first exposed to LED-LCU irradiance during the curing of the bonding agent, posing the highest risk for pulp overheating. Therefore, further studies are needed to address blue and violet light absorption by dentinadhesive interface.

At a distance of $4 \mathrm{~mm}$, wide-spectrum LED-LCUs produced lower pulp chamber temperature increase, though the significance was marginal. There are indications that with increasing distance the LEDLCUs with wider wavelength range result in a more divergent beam ${ }^{40}$. In a recent study which characterized the three-dimensional beam profile of different LEDLCUs it was shown that the Elipar ${ }^{\text {tm }}$ DeepCure (being a narrow-spectrum LED-LCU) had a less divergent beam compared to the Bluephase Style ${ }^{\circledR}$ (wide-spectrum LED-LCU) ${ }^{41}$. Therefore, it is reasonable to believe that with increasing distance the beam of a wide-spectrum LED-LCU compared to a narrow-spectrum LED-LCU would be more divergent resulting in lower temperature increase. This might explain why we observed higher temperature increase for the wide-spectrum LED-LCUs at closer distance ( 0 and $2 \mathrm{~mm}$ ) to the tooth but lower temperature increase at further distance $(4 \mathrm{~mm})$. This may be important in a clinical context, since widespectrum LED-LCUs seem to be more suitable in clinical situations when light guide tip during light-curing is placed further from the pulp.

Irradiance was the strongest predictor for temperature increase on the surface for narrowspectrum LED-LCUs. In our sample two narrowspectrum LED-LCUs, Elipar ${ }^{\text {tm }}$ DeepCure and Elipar ${ }^{\text {tm }}$ S10, had much higher irradiance compared to widespectrum LED-LCUs which could explain why narrowspectrum LED-LCUs had statistically significantly higher temperature increase on the tooth surface. For the wide-spectrum LED-LCUs tested, CT explained most of the temperature increase in the pulp chamber confirming the results from our previous study where only wide-spectrum LED-LCUs were investigated ${ }^{8)}$. For narrow-spectrum LED-LCUs, CT was the dominating factor for pulp chamber temperature and irradiance for surface temperature development.

\section{Effect of output mode}

Importantly, the output mode was the second most dominant factor, both for the pulp chamber and surface temperature development: modulated output mode had a statistically significantly lower temperature increase in the pulp chamber compared to continuous output mode. This finding is in line with other laboratory studies, where 
modulated output mode resulted in lower temperature increase in pulp chamber ${ }^{25,42-45)}$. In addition, the present study showed that modulated output mode had a higher temperature increase on the tooth surface. In situations when the light guide tip is placed close to the pulp, the only one in our sample narrow-spectrum LED-LCU with modulated output mode might be a "safer" option to use in preventing pulp tissue overheating but only when contra-measures for protecting soft tissue, like air-cooling ${ }^{42,46)}$, are used, as this narrow-spectrum LEDLCU with modulated output mode is likely to increase surface temperature more than wide-spectrum LEDLCUs at closer distance.

\section{Effect of light guide tip design}

Light guide tip delivering an inhomogeneous beam was the strongest factor for the surface temperature increase. The present study showed that light guide tip inhomogeneity resulted in higher surface and pulp chamber temperature increase compared to more homogeneous tips. As shown from the results of the liquid crystal sheet test, the Bluephase Style ${ }^{\circledR}$ battery was the only LED-LCU in our study that had an inhomogeneous light guide tip. A laser beam analyzer provides a valid measure of a beam profile, but does not take into consideration the temperature development ${ }^{15)}$. One may assume a correlation between a beam homogeneity and a temperature distribution assessed with the liquid crystal sheet. Several authors have investigated the problem of light guide tip inhomogeneity for LED-LCUs. Some studies suggested that no LED-LCUs provided perfectly homogeneous irradiance distribution ${ }^{28,47}$. This has been shown to lead to both inhomogeneous polymerization ${ }^{48}$ as well as inhomogeneous micro-hardness and elastic modulus distribution of the resin-based composite surface $^{21,49,50)}$. In addition to excessive heat to the exposed oral tissue, the inhomogeneity of the light guide tip can lead to "hot spots" and it has been previously shown that some areas covered by the tip of the light guides can reach more than $2,500 \mathrm{~mW} / \mathrm{cm}^{2}{ }^{51)}$. The manufacturer has since introduced a light guide in newer generation model with a light homogenizer (Bluephase Style ${ }^{\circledR}$ mains powered in our study) that reduced the light beam inhomogeneity. The temperature distribution pattern was also shown to be more homogeneous across its light guide tip when investigated with the liquid crystal sheet.

\section{CONCLUSION}

In conclusion, LED-LCUs with different spectral emission contributed to different temperature development. When compared to the narrow-spectrum LED-LCUs the widespectrum LED-LCUs produced higher pulp chamber temperature at closer distances, but lower temperature at higher distance. Modulated output mode resulted in higher surface temperature increase, but lower pulp chamber temperature increase compared to LED-LCU with continuous output mode. Light guide tip delivering inhomogeneous beam compared to homogeneous beam caused higher pulp chamber and surface temperature increase. Clinicians should be aware that LED-LCUs behave differently for surfaces and pulp chamber temperature development. Therefore, the choice of LED-LCUs may depend on specific clinical situations or specific countermeasures should be applied.

\section{ACKNOWLEDGMENTS}

The study was financially supported by the Norwegian Directorate of Health (14/1493).

\section{CONFLICT OF INTEREST}

The authors declare that they have no conflict of interest.

\section{REFERENCES}

1) Heintze SD, Rousson V. Clinical effectiveness of direct class II restorations - a meta-analysis. J Adhes Dent 2012; 14: 407-431.

2) Santini A, Turner S. General dental practitioners' knowledge of polymerisation of resin-based composite restorations and light curing unit technology. Br Dent J 2011; 211: E13.

3) Kopperud SE, Rukke HV, Kopperud HM, Bruzell EM. Light curing procedures - performance, knowledge level and safety awareness among dentists. J Dent 2017; 58: 67-73.

4) Ernst CP, Price RB, Callaway A, Masek A, Schwarm H, Rullmann I, et al. Visible light curing devices -irradiance and use in 302 german dental offices. J Adhes Dent 2018: $1-15$.

5) Durner J, Obermaier J, Draenert M, Ilie N. Correlation of the degree of conversion with the amount of elutable substances in nano-hybrid dental composites. Dent Mater 2012; 28: 11461153.

6) Ferracane JL, Mitchem JC, Condon JR, Todd R. Wear and marginal breakdown of composites with various degrees of cure. J Dent Res 1997; 76: 1508-1516.

7) Leprince J, Devaux J, Mullier T, Vreven J, Leloup G. Pulpaltemperature rise and polymerization efficiency of LED curing lights. Oper Dent 2010; 35: 220-230.

8) Mouhat M, Mercer J, Stangvaltaite L, Ortengren U. Lightcuring units used in dentistry: factors associated with heat development-potential risk for patients. Clin Oral Investig 2017; 21: 1687-1696.

9) Santini A, Watterson C, Miletic V. Temperature rise within the pulp chamber during composite resin polymerisation using three different light sources. Open Dent J 2008; 2: 137141.

10) Runnacles P, Arrais CA, Pochapski MT, Dos Santos FA, Coelho U, Gomes JC, et al. In vivo temperature rise in anesthetized human pulp during exposure to a polywave LED light curing unit. Dent Mater 2015; 31: 505-513.

11) Zach L, Cohen G. Pulp response to externally applied heat. Oral Surg Oral Med Oral Pathol 1965; 19: 515-530.

12) Spranley TJ, Winkler M, Dagate J, Oncale D, Strother E. Curing light burns. Gen Dent 2012; 60: e210-214.

13) Maucoski C, Zarpellon DC, Dos Santos FA, Lipinski LC, Campagnoli EB, Rueggeberg FA, et al. Analysis of temperature increase in swine gingiva after exposure to a Polywave((R)) LED light curing unit. Dent Mater 2017; 33: 1266-1273.

14) Jakubinek MB, O’Neill C, Felix C, Price RB, White MA. Temperature excursions at the pulp-dentin junction during the curing of light-activated dental restorations. Dent Mater 2008; 24: 1468-1476.

15) Rueggeberg FA, Giannini M, Arrais CAG, Price RB. Light curing in dentistry and clinical implications: a literature 
review. Braz Oral Res 2017; 31(suppl 1): e61.

16) Price RB, Ferracane JL, Shortall AC. Light-curing units: A review of what we need to know. J Dent Res 2015; 94: 11791186.

17) Park SH, Roulet JF, Heintze SD. Parameters influencing increase in pulp chamber temperature with light-curing devices: curing lights and pulpal flow rates. Oper Dent 2010; 35: 353-361.

18) Harlow JE, Rueggeberg FA, Labrie D, Sullivan B, Price RB. Transmission of violet and blue light through conventional (layered) and bulk cured resin-based composites. J Dent 2016; 53: 44-50.

19) Jandt KD, Mills RW. A brief history of LED photopolymerization. Dent Mater 2013; 29: 605-617.

20) Santini A, Miletic V, Swift MD, Bradley M. Degree of conversion and microhardness of TPO-containing resin-based composites cured by polywave and monowave LED units. J Dent 2012; 40: 577-584.

21) Haenel T, Hausnerova B, Steinhaus J, Price RB, Sullivan B, Moeginger B. Effect of the irradiance distribution from light curing units on the local micro-hardness of the surface of dental resins. Dent Mater 2015; 31: 93-104.

22) Price RB, Labrie D, Rueggeberg FA, Felix CM. Irradiance differences in the violet $(405 \mathrm{~nm})$ and blue $(460 \mathrm{~nm})$ spectral ranges among dental light-curing units. J Esthet Restor Dent 2010; 22: 363-377.

23) Sudheer V, Manjunath M. Contemporary curing profiles: Study of effectiveness of cure and polymerization shrinkage of composite resins: An in vitro study. J Conserv Dent 2011; 14: 383-386.

24) Harlow JE, Sullivan B, Shortall AC, Labrie D, Price RB. Characterizing the output settings of dental curing lights. J Dent 2016; 44: 20-26.

25) Huang TK, Hung CC, Tsai CC. Reducing, by pulse width modulation, the curing temperature of a prototype highpower LED light curing unit. Dent Mater J 2006; 25: 309315.

26) Michaud PL, Price RB, Labrie D, Rueggeberg FA, Sullivan B. Localised irradiance distribution found in dental light curing units. J Dent 2014; 42: 129-139.

27) Sampaio CS, Atria PJ, Rueggeberg FA, Yamaguchi S, Giannini M, Coelho PG, et al. Effect of blue and violet light on polymerization shrinkage vectors of a CQ/TPO-containing composite. Dent Mater 2017; 33: 796-804.

28) Price RB, Rueggeberg FA, Labrie D, Felix CM. Irradiance uniformity and distribution from dental light curing units. $J$ Esthet Restor Dent 2010; 22: 86-101.

29) The American heritage science dictionary. Houghton Mifflin Company; 2005. Liquid crystal; available from: https://ahdictionary.com/word/search. html?q=liquid+crystal+display.

30) Iijima T, Zhang JQ. Three-dimensional wall structure and the innervation of dental pulp blood vessels. Microsc Res Tech 2002; 56: 32-41.

31) Ahn J, Pogrel MA. The effects of $2 \%$ lidocaine with 1:100,000 epinephrine on pulpal and gingival blood flow. Oral Surg Oral Med Oral Pathol Oral Radiol Endod 1998; 85: 197-202.

32) Runnacles P, Arrais CAG, Maucoski C, Coelho U, De Goes MF, Rueggeberg FA. Comparison of in vivo and in vitro models to evaluate pulp temperature rise during exposure to a Polywave(R) LED light curing unit. J Appl Oral Sci 2019; 27: e20180480.
33) Data G. Dental Light Cure Equipment - Dental Market Analysis and Forecast Model. 2018.

34) Baldissara P, Catapano S, Scotti R. Clinical and histological evaluation of thermal injury thresholds in human teeth: a preliminary study. J Oral Rehabil 1997; 24: 791-801.

35) Pohto M, Scheinin A. Microscopic observations on living dental pulp II. The effect of thermal irritants on the circulation of the pulp in the lower rat incisor. Acta Odontol Scand 1958; 16: 315-327.

36) Price RB, Shortall AC, Palin WM. Contemporary issues in light curing. Oper Dent 2014; 39: 4-14.

37) Uusitalo E, Varrela J, Lassila L, Vallittu PK. Transmission of curing light through moist, air-dried, and EDTA treated dentine and enamel. BioMed Res int 2016; 2016: 5713962.

38) Shimokawa C, Sullivan B, Turbino ML, Soares CJ, Price RB. Influence of emission spectrum and irradiance on light curing of resin-based composites. Oper Dent 2017; 42: 537-547.

39) Par M, Repusic I, Skenderovic H, Tarle Z. Wavelengthdependent light transmittance in resin composites: practical implications for curing units with different emission spectra. Clin Oral Investig 2019; 23: 4399-4409.

40) Price RB, Labrie D, Whalen JM, Felix CM. Effect of distance on irradiance and beam homogeneity from 4 light-emitting diode curing units. J Can Dent Assoc 2011; 77: b9.

41) Juckes S, Sullivan B, Kostylev I, Price R, Labrie D. Threedimensional beam profiling used to characterize dental lightcuring units. Appl Opt 2019; 58: 9540-9547.

42) Onisor I, Asmussen E, Krejci I. Temperature rise during photo-polymerization for onlay luting. Am J Dent 2011; 24: 250-256.

43) Lu H, Stansbury JW, Bowman CN. Impact of curing protocol on conversion and shrinkage stress. J Dent Res 2005; 84: 822 826.

44) Hubbezoglu I, Dogan A, Dogan OM, Bolayir G, Bek B. Effects of light curing modes and resin composites on temperature rise under human dentin: An in vitro study. Dent Mater J 2008; 27: 581-589.

45) Jo SA, Lee CH, Kim MJ, Ferracane J, Lee IB. Effect of pulsewidth-modulated LED light on the temperature change of composite in tooth cavities. Dent Mater 2019; 35: 554-563.

46) Zarpellon DC, Runnacles P, Maucoski C, Coelho U, Rueggeberg FA, Arrais C. Controlling in vivo, human pulp temperature rise caused by LED curing light exposure. Oper Dent 2019; 44: 235-241.

47) Al-Zain AO, Eckert GJ, Lukic H, Megremis S, Platt JA. Polymerization pattern characterization within a resin-based composite cured using different curing units at two distances. Clin Oral Investig 2019; 23: 3995-4010.

48) Arikawa H, Kanie T, Fujii K, Takahashi H, Ban S. Effect of inhomogeneity of light from light curing units on the surface hardness of composite resin. Dent Mater J 2008; 27: 21-28.

49) Price RB, Labrie D, Rueggeberg FA, Sullivan B, Kostylev I, Fahey J. Correlation between the beam profile from a curing light and the microhardness of four resins. Dent Mater 2014; 30: 1345-1357.

50) Issa Y, Watts DC, Boyd D, Price RB. Effect of curing light emission spectrum on the nanohardness and elastic modulus of two bulk-fill resin composites. Dent Mater 2016; 32: 535550.

51) Shortall AC, Price RB, MacKenzie L, Burke FJ. Guidelines for the selection, use, and maintenance of LED light-curing units -Part 1. Br Dent J 2016; 221: 453-460. 